\title{
Search for p-mode oscillations in RX $\mathrm{J} 2117.1+3412^{\star}$
}

\author{
H.-K. Chang ${ }^{1,2}$, I.-C. Shih ${ }^{3}$, C.-Y. Liu ${ }^{2,3}$, T. Fan ${ }^{1}$, Y.-S. Wu ${ }^{1}$, F. Roques ${ }^{3}$, A. Doressoundiram ${ }^{3}$, A. Fernandez ${ }^{3}$, \\ B. Christophe ${ }^{3}$, and F. Dauny ${ }^{3}$
}

\author{
1 Institute of Astronomy, National Tsing Hua University, 30013 Hsinchu, Taiwan \\ e-mail: hkchang@phys.nthu.edu.tw \\ 2 Department of Physics, National Tsing Hua University, 30013 Hsinchu, Taiwan \\ 3 LESIA, Paris Observatory, 92195 Meudon, France
}

Received 20 June 2013 / Accepted 13 August 2013

\begin{abstract}
We report the effort to search for white dwarf p-mode oscillations in RX J2117.1+3412 (V2027 Cyg), a GW Vir star. The data were taken with Miosotys, a multi-fiber photometer, mounted on the 1.93-m telescope at Observatoire de Haute-Provence (OHP) with a 20-Hz cadence in May 2010 and June 2012. Two intriguing signatures at $1.2081 \mathrm{~Hz}$ and $2.5852 \mathrm{~Hz}$ were found at 3.8- $\sigma$ and 3.5- $\sigma$ significance levels, respectively. However, possible atmospheric effects cannot be ruled out. If these two features are not real, the 3- $\sigma$ upper limit of the relative amplitude for the possible oscillations in the frequency range from 1 to $10 \mathrm{~Hz}$ is estimated to be about $6 \times 10^{-4}$. This is the first report in the literature for a p-mode oscillation search of GW Vir stars.
\end{abstract}

Key words. stars: individual: RX J2117.1+3412 - stars: oscillations - white dwarfs

\section{Introduction}

Asteroseismology of white dwarfs, which started from the discovery of the first pulsating DA white dwarf, HL Tau 76 (Landolt 1968), has brought tremendous progress in our understanding of white dwarf structure and evolution. For a comprehensive review, readers are referred to Fontaine \& Brassard (2008) and Winget \& Kepler (2008). Along the white dwarf cooling sequence, there are three groups of pulsating white dwarfs: GW Vir stars (DOVs, or pulsating PG 1159 stars, which have a helium/carbon/oxygen atmosphere and effective temperature around $120000 \mathrm{~K}$ ), V777 Her stars (DBVs, which have a helium-dominated atmosphere and effective temperature around $25000 \mathrm{~K}$ ), and $\mathrm{ZZ}$ Ceti stars (DAVs, which have a hydrogen-dominated atmosphere and effective temperature around $12000 \mathrm{~K})$. A fourth group, the pulsating Hot DQ white dwarfs (which have a carbon-dominated atmosphere and effective temperature around $20000 \mathrm{~K}$ ) was identified recently (Montgomery et al. 2008; Dufour et al. 2011). With the discovery of pulsations in the extremely-low-mass white dwarf SDSS J184037.78+642312.3 (Hermes et al. 2012), which has a mass of $0.17 M_{\odot}$, effective temperature of about $9000 \mathrm{~K}$, and a hydrogen-dominated atmosphere, another new class of white dwarf pulsators has likely emerged. Oscillations detected in these white dwarfs have periods ranging from $100 \mathrm{~s}$ to $5000 \mathrm{~s}$. They are all well interpreted as g-mode oscillations driven by the $\kappa$-mechanism due to partial ionization of the main envolpe constituents (e.g. Fontaine \& Brassard 2008, and references therein). From these measured oscillation periods, very accurate inference of the values of white dwarf mass, rotation period, and outer-layer thickness can be made.

On the other hand, white dwarf p-mode oscillations have not yet been detected. These oscillations have periods shorter than

\footnotetext{
* Reduced light curves are only available at the CDS via anonymous ftp to cdsarc.u-strasbg.fr (130.79.128.5) or via http://cdsarc.u-strasbg.fr/viz-bin/qcat?]/A+A/558/A63
}

g-mode ones. Contrary to solar-like stars, p-mode oscillations in white dwarfs propagate mainly in the interior. Their properties, such as periods, are therefore more sensitive to the internal structure and constituents of a white dwarf. Detection of p-mode oscillations definitely brings more information and insight to the study of white dwarfs.

To date, efforts to search for white dwarf p-mode oscillations have been made only for DA and DB white dwarfs. In Robinson(1984), upper limits to the p-mode amplitude of 19 DA white dwarfs were reported. These upper limits are mostly between $0.5 \times 10^{-3}$ and $2 \times 10^{-3}$ (relative amplitude) with Nyquist frequencies between 5 and $10 \mathrm{~Hz}$ or so. Five of these 19 targets were observed with the 2.1-m McDonald telescope, and the other 14 were taken from earlier surveys with smaller telescopes. Two DB white dwarfs, GD 358 and PG 0112+104, were observed with the Hubble Space Telescope (Kawaler et al. 1994). No evidence of pulsations was found in the frequency range from 1 to $12 \mathrm{~Hz}$. The estimated upper limit for GD 358 is at the 0.0016 mag level and two times larger for PG 0112+104. A more recent effort to search for p-mode oscillations in nine DA white dwarfs with the VLT-ULTRACAM was reported in Silvotti et al. (2011). Inferred upper limits (relative amplitude) are typically between $2 \times 10^{-4}$ and $8 \times 10^{-4}$ for six of the nine targets in the frequency range from $0.1 \mathrm{~Hz}$ to the respective Nyquist frequencies, which are about 10 and $45 \mathrm{~Hz}$. The other three targets have upper limits at about $1 \times 10^{-3}-3 \times 10^{-3}$.

We report the result of a p-mode oscillation search in the hottest pulsating PG 1159 star, RX J2117.1+3412, which is obtained from observations conducted in May 2010 and June 2012 using MIOSOTYS (Shih et al. 2010) mounted on the 1.93-m telescope at Observatoire de Haute-Provence (OHP) in southern France. Although radial oscillations of white dwarfs were suggested a long time ago (e.g. Ostriker 1971) and their excitation was expected from calculation with relatively realistic DA and DB white dwarf models (Saio et al. 1983; Starrfield et al. 1983; Kawaler 1993), detailed quantitative calculation 

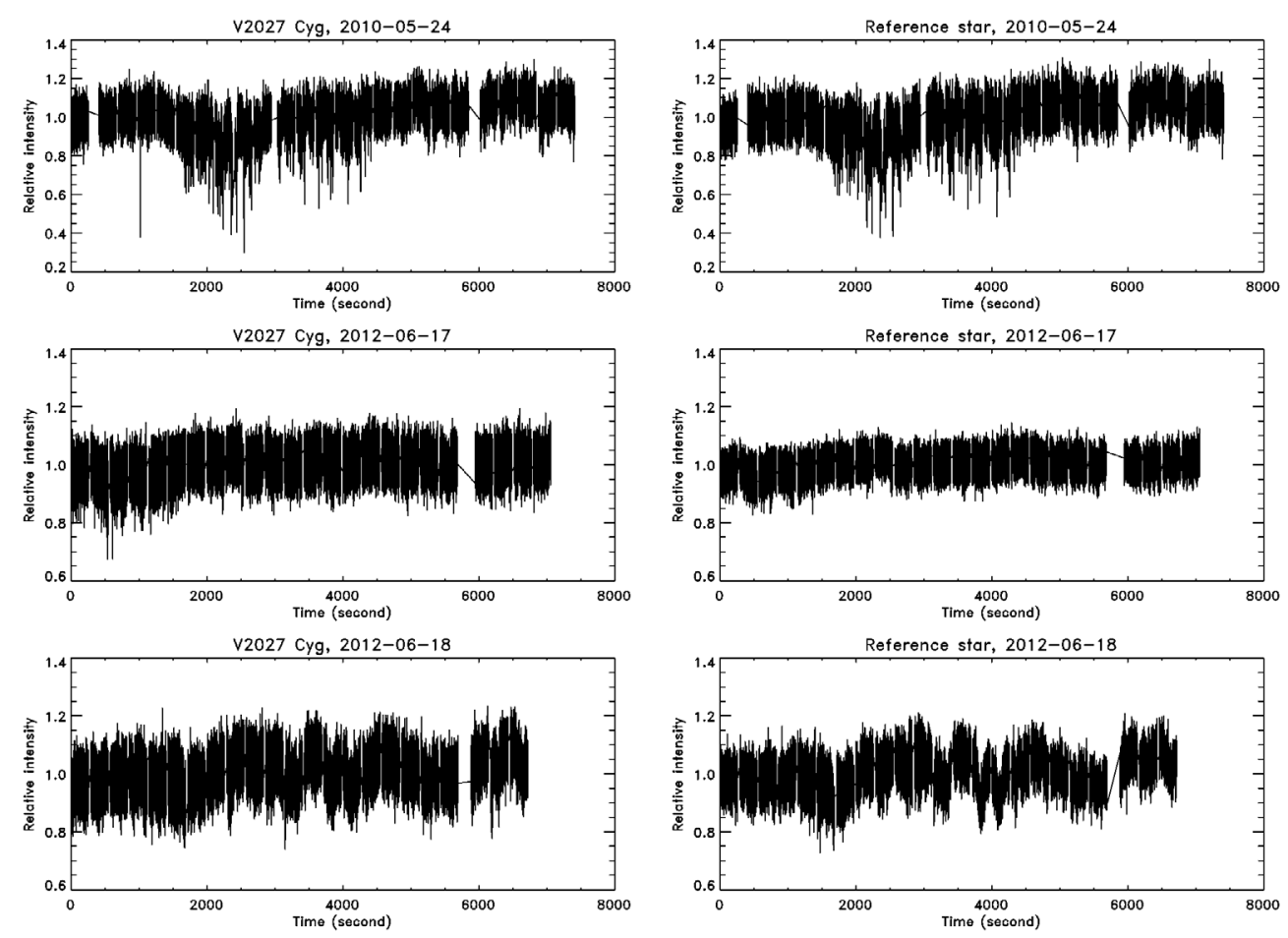

Fig. 1. Light curves of V2027 Cyg and reference stars taken by Miosotys on May 24, 2010, June 17, 2012, and June 18, 2012. See the main text for more details about the data. The dip down to about 0.4 relative intensity at the left of the top left panel may be a TNO occultation event, which does not affect our analysis in this work. Other spiky dips are apparently due to atmospheric effects.

for p-mode excitation in PG 1159 stars seems lacking. On the other hand, the driving mechanism of g-mode oscillations in PG 1159 stars has been investigated in Quirion et al. (2004) and Gautschy et al. (2005). Quite extensive asteroseismic inferences of some GW Vir stars that have observed g-mode oscillations can be found in Kawaler et al. (1994) and Corsico \& Althaus (2006). A detailed description of observed g-mode oscillations in RX J2117.1+3412 and their inferences are reported in Vauclair et al. (2002).

The object RX J2117.1+3412 was discovered in the ROSAT sky survey and identified as a PG 1159 star (Motch et al. 1993). More spectral analysis indicates that its effective temperature is about $170000 \mathrm{~K}$, and its surface gravity $g$ is $\log g=6.0_{-0.2}^{+0.3}$ (Gaussian units) (Werner et al. 1996; Rauch $\&$ Werner 1997). From identified g-mode oscillation periods of RX J2117.1+3412, its mass is estimated to be $0.56_{-0.04}^{+0.02} M_{\odot}$, and its rotation period is $1.16 \pm 0.05$ days. However, its rotational splitting of measured periods decreases with increasing periods. It is inconsistent with a solid body rotation (Vauclair et al. 2002). This suggests the presence of differential rotation, which apparently also exists in the interior. Since p-mode oscillations are more sensitive to internal structure, detection of these modes is highly desired.

In the following, we describe the observation and data reduction in Sect. 2, and the analysis to search for periodicity in Sect. 3. The summary and discussion are in Sect. 4.

\section{Observations and data reduction}

The star RX J2117.1+3412 was observed using one of the 30 fibers of the Miosotys instrument mounted on the 1.93-m telescope at OHP in May 2010 and June 2012 for several nights. The main purpose of Miosotys (Shih et al. 2010) is to search for serendipitous trans-Neptunian object (TNO) occultation events. Its current data-acquisition system yields a series of data cubes with no attempt to ensure the accuracy of absolute timing. Each data cube consists of 5120 successive frames at a cadence of $20 \mathrm{~Hz}$. Each frame records the 30 images of the sky areas covered by the fibers at the telescope focal plane. Time gaps between data cubes can be half a minute or longer for different reasons.

Each fiber covers a $12^{\prime \prime} \times 12^{\prime \prime}$ field of view, whose image is recorded in the detector with an area of $80 \times 80$ pixels. The star RX J2117.1+3412 and the chosen reference stars discussed below are the only visible targets in each field. Thus, simple stellar aperture photometry was performed to derive the light curves. Due to the electronic characteristics of the EMCCD camera at a high-speed readout, which introduces unstable and widevariance bias levels, conventional CCD bias treatment to the images actually worsens the signal-to-noise ratio of the data. Using the unexposed CCD-pixel regions outside the aforementioned 30 images in each frame, we developed an alternative treatment to properly deal with the bias level (Shih et al., in prep.).

Data quality of some of those nights is not good due to weather conditions. We decided to use the data acquired on May 24, 2010, June 17, 2012, and June 18, 2012. We choose reference stars as close as possible to the target star, RX J2117.1+3412 (V2027 Cyg). Since the original purpose of the observation is for a TNO occultation search, fiber locations are arranged for stars suitable for that purpose. The closest star for the observation on May 24, 2010 is a star about two arcminutes to the north of V2027 Cyg and a star about one arcminute to the south-east for the observations on June 17, 2012 and June 18, 2012. The light curves of V2027 Cyg and reference stars are shown in Fig. 1.

Because of the significant angular distance between the target and reference stars, they may experience the same atmopheric variation at different times or simply different atmopheric variations. A normalized light curve obtained by taking the ratio of the target star light curve to the reference one is not useful. In 

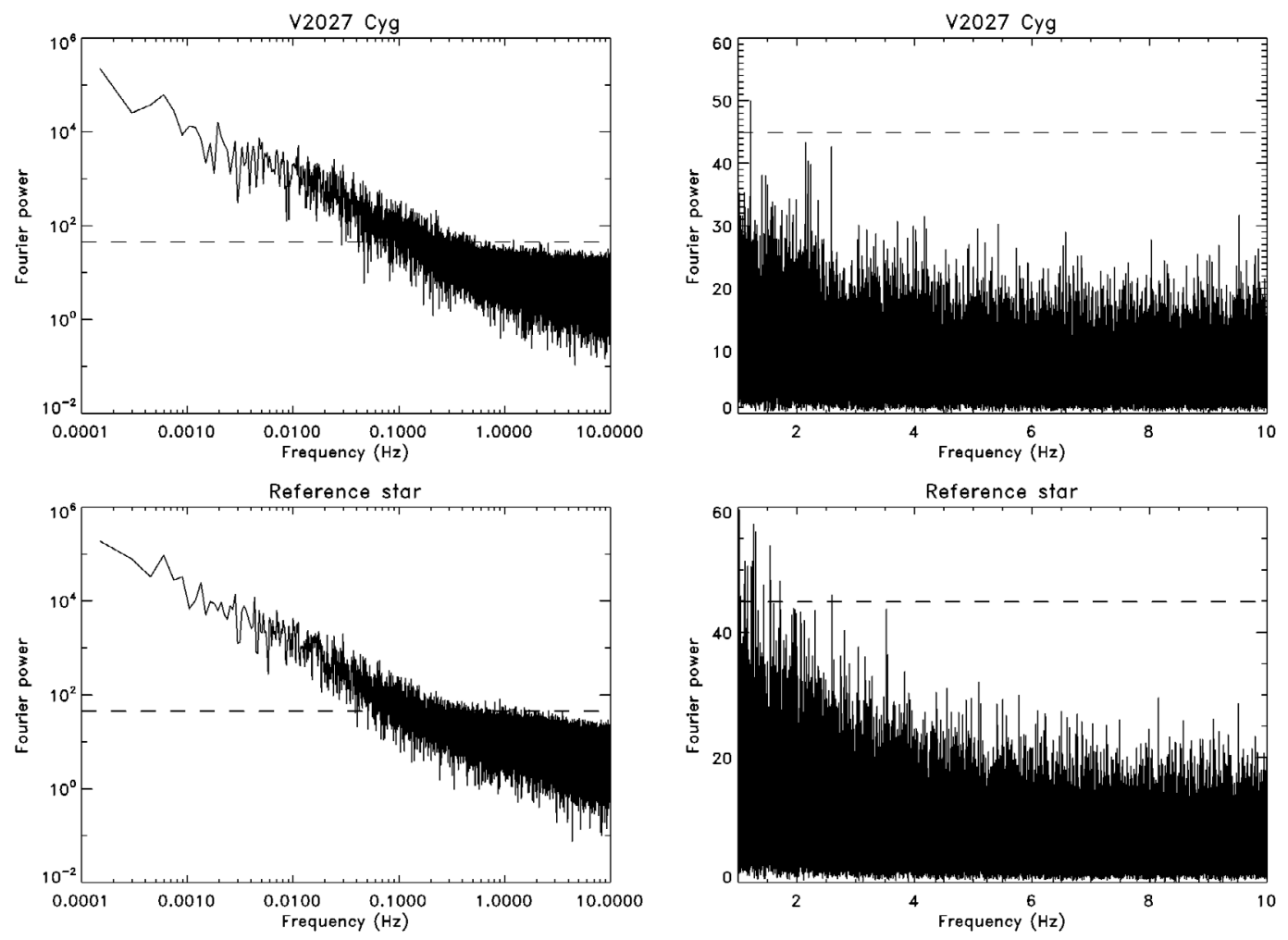

Fig. 2. Combined Fourier power spectra from the 3 data sets. The Fourier power of a data set is defined in Sect. 3 . The dashed lines in all the panels mark the 3- $\sigma$ level above which the random probability is lower than $0.3 \%$, considering the number of independent trials of about 60000 . Left panels are plotted in a log-log scale to show the high power at low frequencies, which are due to atmospheric variations. Right panels show the power spectra in $1-10 \mathrm{~Hz}$.

the following analysis, we compare the Fourier power spectra of the target and reference stars for more discussion.

\section{Periodicity search}

We fill the gaps in the light curves with a constant flux, so that the two-hour data sets at each of the three nights can be used to search for periodicity with a Fourier transform. The discrete Fourier transform was calculated for the three data sets separately. To facilitate combination and comparison of results of these three data sets, we employed the same amount of data length for all the three nights, which is 134240 frames at $20 \mathrm{~Hz}$. The summed Fourier power spectrum of these three data sets is shown in Fig. 2.

For the convenience of the discussion, we include the Fourier transform formula in the following to clearly show the magnitude of the Fourier power. The Fourier power we adopt is defined as (van der Klis 1989)

$P_{j}=\frac{2}{N_{\mathrm{t}}}\left|a_{j}\right|^{2}$

where $j=0,1, \ldots, \frac{N}{2}, N$ is the number of data points, $N_{\mathrm{t}}$ is the number of total counts, and

$a_{j}=\sum_{k=0}^{N-1} x_{k} \mathrm{e}^{i\left(2 \pi j \frac{k}{N}\right)}$,

with $x_{k}$ being the measured counts at the $k$ th data point. The Fourier power of random noise follows the $\chi^{2}$ distribution with two degrees of freedom if the $x_{k}$ follows a Gaussian distribution, and the normalization in Eq. (1) is chosen so that the average noise power is $\left\langle P_{j}\right\rangle_{j \neq 0}=2$ if $x_{k}$ follows the Poisson distribution (van der Klis 1989).

In our data, the dark current and bias are negligible. The readings recorded are essentially the photon counts multiplied by an amplification factor. To assess the random probability of possible features in the power spectrum when conducting a discrete Fourier transform on a data set, we always renormalize the Fourier power by a factor, so that the average power from $8 \mathrm{~Hz}$ to $10 \mathrm{~Hz}$ is equal to 2 . We choose this frequency range to avoid the power of lower-frequency atmospheric variations.

For the analysis with data of each individual night, we present our results in terms of the Fourier relative amplitude, which is (van der Klis 1989)

$r \equiv \frac{\sqrt{\sigma_{x}^{2}}}{\bar{x}}=\sqrt{\frac{\sum_{j=1}^{\frac{N}{2}-1} P_{j}+\frac{1}{2} P_{\frac{N}{2}}}{N_{\mathrm{t}}}}$,

where $\sigma_{x}^{2}$ is the variance of the data and $\bar{x}$ is the average. We present the relative amplitude at the $j$ th frequency as $r_{j}=$ $\sqrt{P_{j} / N_{\mathrm{t}}}$ for $j \neq \frac{N}{2}$. The amplification factor mentioned above is cancelled out in this relative amplitude expression. We note that to enhance possibly existing weak signals, one can add the Fourier power of different data sets, as defined above, and use the $\chi^{2}$ distribution statistics to evaluate significance levels of possible signals, if the data follows a Gaussian distribution in nature.

In Fig. 2, we can see that the high power at frequencies lower than $1 \mathrm{~Hz}$ is most likely due to atmospheric variations since they appear in both the target and reference stars in a similar way. We are not able to proceed to discuss any possible periodicity 

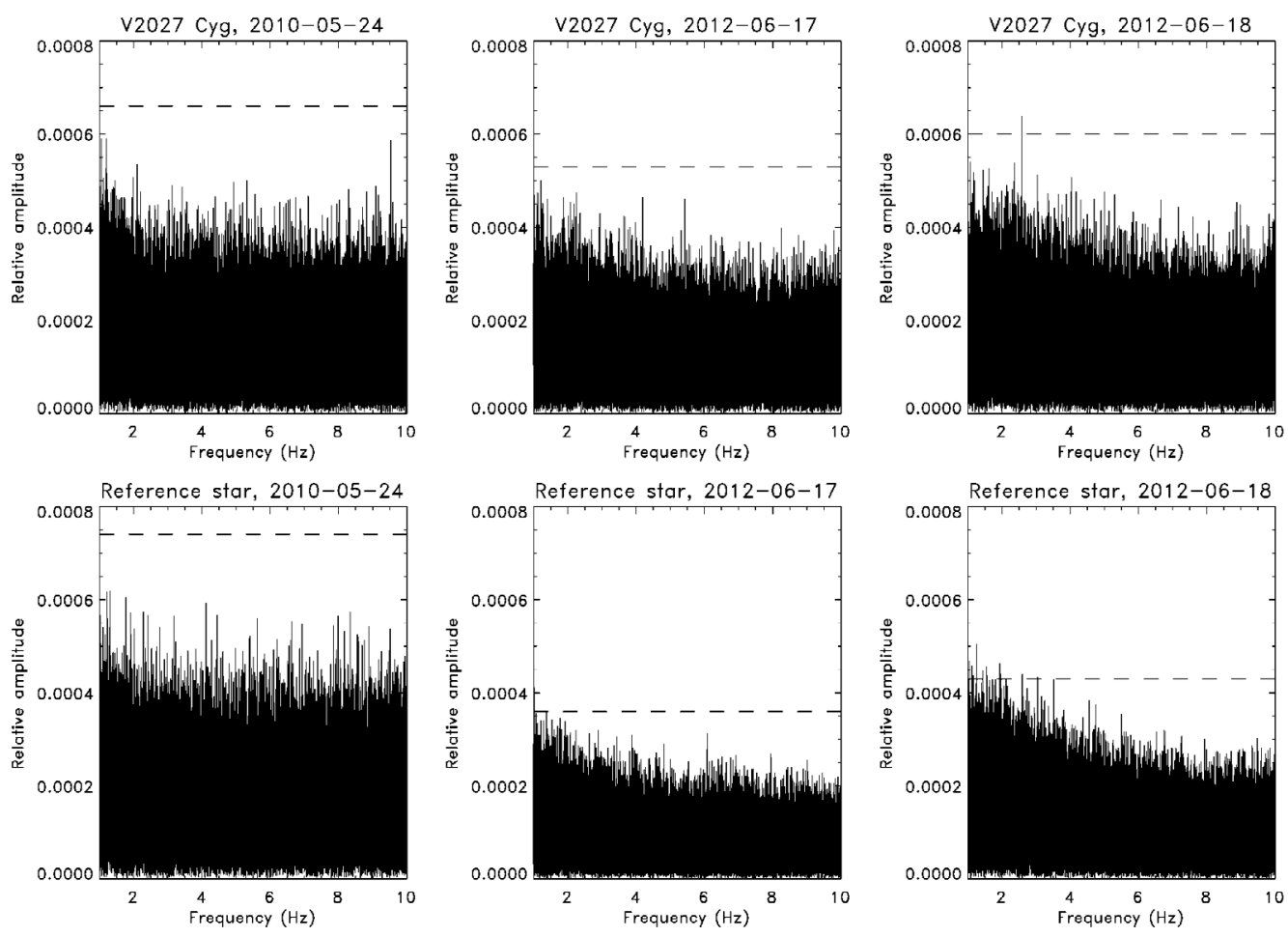

Fig. 3. Fourier relative amplitude spectra of V2027 Cyg and the reference star with data taken on May 24, 2010, June 17, 2012 , and June 18, 2012. The dashed lines mark the 3- $\sigma$ level. For the data of June 17 and June 18, 2012, the reference star has lower relative amplitudes (lower middle and lower right panels) because of a larger measured flux. One prominent feature appears at $2.5852 \mathrm{~Hz}$ in the upper right panel. It does not have a corresponding feature in the lower right panel, in which a seemingly close peak at about the 3- $\sigma$ significance level is actually at $2.5954 \mathrm{~Hz}$. The frequency resolution in this analysis is about $1.5 \times 10^{-4} \mathrm{~Hz}$.

signatures at those frequencies. At frequencies higher than $1 \mathrm{~Hz}$, there is one feature standing out beyond the 3- $\sigma$ level and another two below this level. We note that the $\chi^{2}$ distribution is additive and the sum of the three Fourier power spectra simply follows a $\chi^{2}$ distribution with 6 degrees of freedom if there is only random noise in the data. In all the power spectrum figures, a 3- $\sigma$ confidence level is plotted. The random probability for features higher than this level is lower than $0.3 \%$, considering the number of independent trails, which is about 60000 . One cannot rule out the possibility that these three features are of atmospheric origin if not due to random fluctuations since there are many peaks around this frequency range in the power spectrum of the reference star.

Although the coherence time of observed g-mode oscillations is long, their amplitude could be variable (Vauclair et al. 2002). It is likely the same for p-mode ones. We therefore also examine the power spectrum for each night separately. In Fig. 3, the Fourier relative amplitude spectra of the target and reference stars are shown. Among all the data sets, only one feature has a significance level higher than $3-\sigma$. It is at $2.5852 \mathrm{~Hz}$ in the data of June 18,2012 . Its random probability is about $2.6 \times 10^{-4}$ based on the $\chi^{2}$ distribution (about 3.5- $\sigma$ significance in terms of Gaussian statistics). The corresponding relative amplitude is $6.4 \times 10^{-4}$. The veracity of this feature is jeopardized by the appearance of many peaks in the power spectrum of the reference star around that frequency and below. It is then possibly due to atmospheric variations, although it is prominent in the amplitude spectrum of the target star. This $2.5852-\mathrm{Hz}$ feature does not show up in the data of June 17, 2012 or of May 24, 2010, and its significance is reduced to be at just about $3-\sigma$ in the summed power spectrum of data of June 17 and 18, as seen in Fig. 4, and below $3-\sigma$, as seen in Fig. 2. On the other hand, another feature
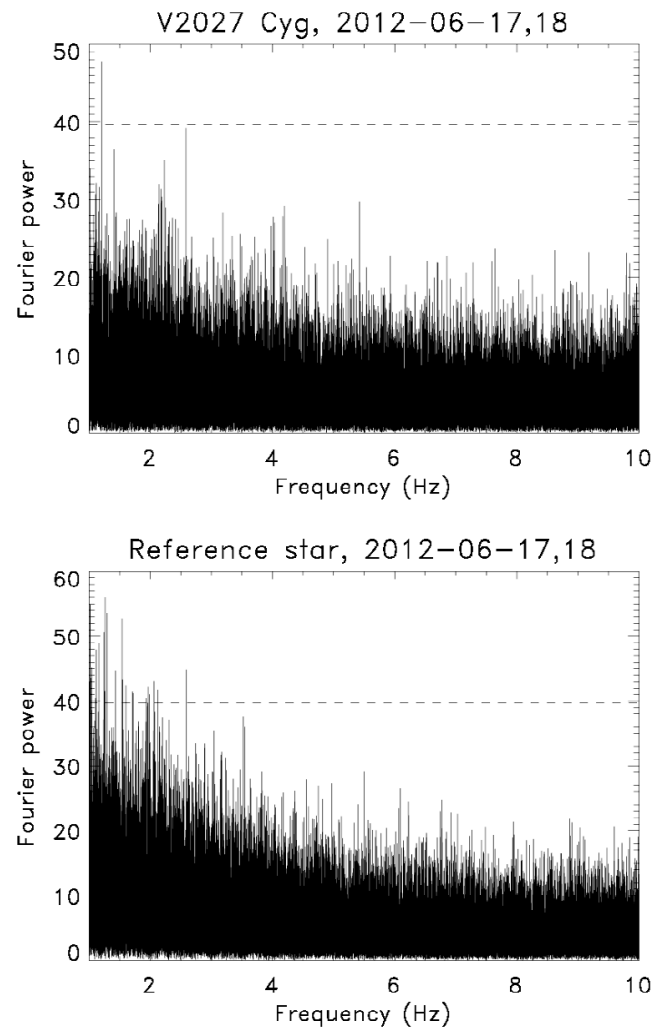

Fig. 4. Combined Fourier power spectrum of data taken on June 17 and 18,2012 . The dashed lines mark the $3-\sigma$ level. In the upper panel, a new signature well above the $3-\sigma$ level appears at $1.2081 \mathrm{~Hz}$, while the significance of the $2.5852 \mathrm{~Hz}$ one (the upper right panel in Fig. 3) decreases. 
Table 1. Fourier power of the five highest features in the top right panel in Fig. 2.

\begin{tabular}{cccccc}
\hline \hline \multirow{2}{*}{ Frequency } & Reference star & \multicolumn{5}{c}{ V2027 Cyg } \\
\cline { 3 - 6 } & 3-night sum & 3-night sum & May 24, 2010 & June 17, 2012 & June 18, 2012 \\
\hline $1.2081 \mathrm{~Hz}$ & 14.68 & 49.97 & 2.257 & 30.31 & 17.40 \\
$2.1441 \mathrm{~Hz}$ & 12.10 & 43.31 & 11.29 & 19.95 & 12.07 \\
$2.1886 \mathrm{~Hz}$ & 5.456 & 40.32 & 9.884 & 13.77 & 16.67 \\
$2.2326 \mathrm{~Hz}$ & 6.327 & 39.81 & 4.718 & 21.50 & 13.59 \\
$2.5852 \mathrm{~Hz}$ & 7.779 & 42.63 & 3.385 & 0.718 & 38.53 \\
\hline
\end{tabular}
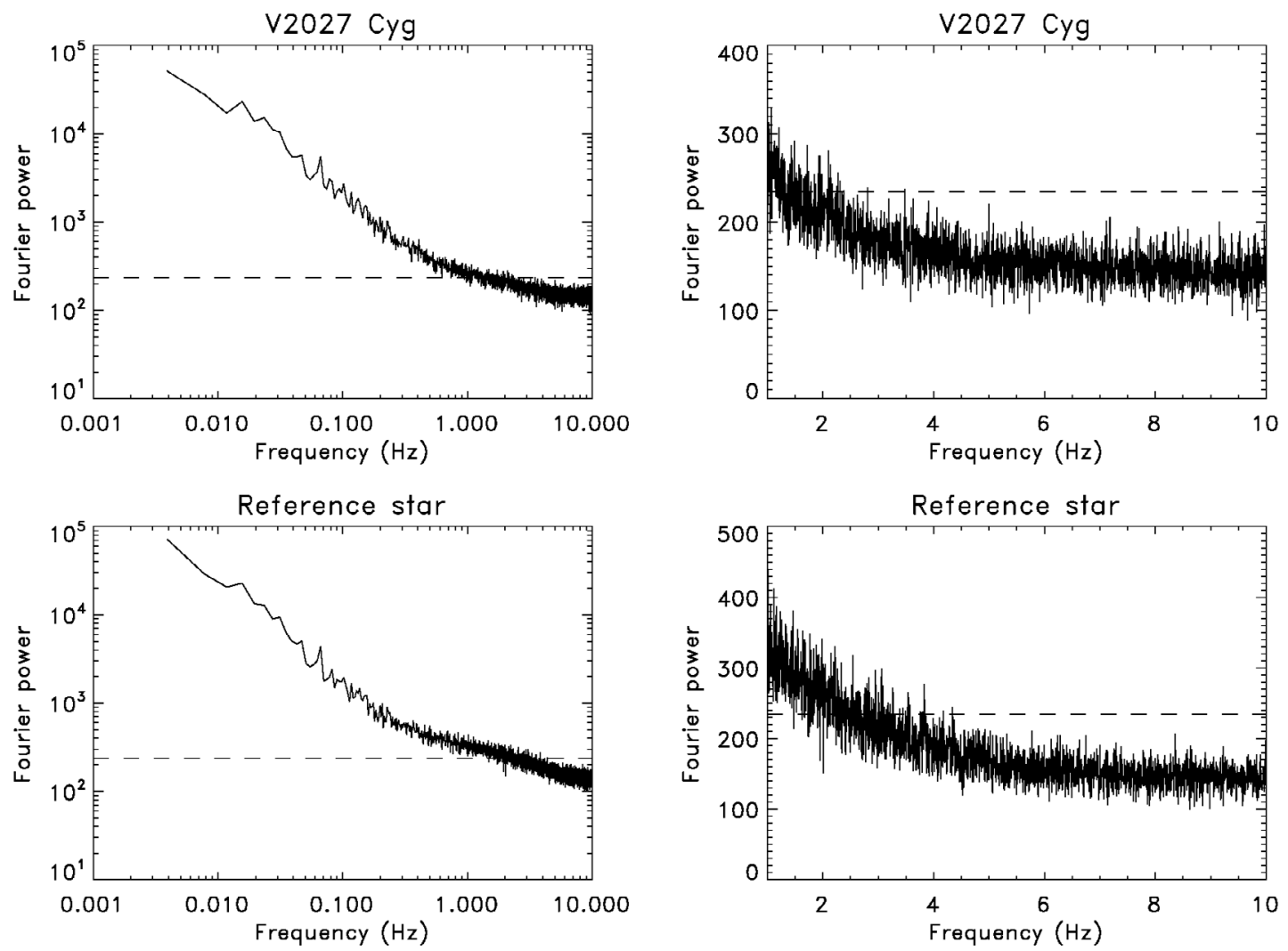

Fig. 5. Combined Fourier power spectra from all the 72 data cubes taken from the three nights. The length of each cube is $256 \mathrm{~s}$. The shorter data length seems to be the reason that atmospheric effects affect the power spectrum up to a higher frequency than that seen in Fig. 2. Dashed lines mark the 3- $\sigma$ level.

at $1.2081 \mathrm{~Hz}$ of random probability about $6.4 \times 10^{-5}$ (i.e., at about 3.8- $\sigma$ significance) emerges in Fig. 4. Its relative amplitude averaged over the two nights is $4.7 \times 10^{-4}$. This feature is present in the data of June 17 and 18, 2012 with moderate significance but is not present in that of May 24, 2010. It therefore appears in Fig. 2 at about 3.45- $\sigma$ significance. If we again compare that to the reference star power spectrum in Fig. 4, the possibility for the $1.2081-\mathrm{Hz}$ feature being due to atmospheric variations seems high.

In Table 1, we summarize the Fourier power of the five features with the highest Fourier power in the top right panel in Fig. 2. Their Fourier power value for the 3-night combined one is shown in the $3 \mathrm{rd}$ column. That of the reference stars at the same frequencies are listed in the 2 nd column. The 4th, 5th and 6th columns show the power value for data of each individual night. The Fourier power of a data set follows a $\chi^{2}$ distribution with 2 degrees of freedom if there is only random noise. Considering the number of independent trials, which is about 60000 in this analysis, the so-called 3- $\sigma$ level, above which the random probability is smaller than $3 \%$, is at the Fourier power equal to 33.6. Those summed Fourier power over
3 nights follow a $\chi^{2}$ distribution with 6 degrees of freedom. The corresponding 3- $\sigma$ level is at power equal to 44.9. One can see from this table that the $1.2081-\mathrm{Hz}$ signature appears in both data sets of June 17 and 18, 2012, but with modest significance. The 2.5852-Hz one appears only on June 18, 2012.

If the oscillation coherence time is shorter than the length of the data set we used, such as in the current case of $2 \mathrm{~h}$ or so, their periodcity signature is smeared out in the analysis mentioned above. We therefore also conducted Fourier transform on each cube of the data to search for oscillations with a coherence time shorter than $2 \mathrm{~h}$ but longer than $256 \mathrm{~s}$, which is the length of each data cube. The summed power spectrum of the 72 data cubes is shown in Fig. 5. The atmospheric variation affects the power spectrum up to a higher frequency than that of Fig. 2 because of the much shorter length of the data cube. In this analysis, we did not find any interesting features in the summed power spectrum of the 72 data cubes or in the spectra of each individual nights separately. The signatures at $1.2081 \mathrm{~Hz}$ and $2.5852 \mathrm{~Hz}$ discussed above do not show up in Fig. 5. This is consistent, because the corresponding relative amplitude of the noise level in Fig. 5 is about $7 \times 10^{-4}$, which is already higher than that associated with 

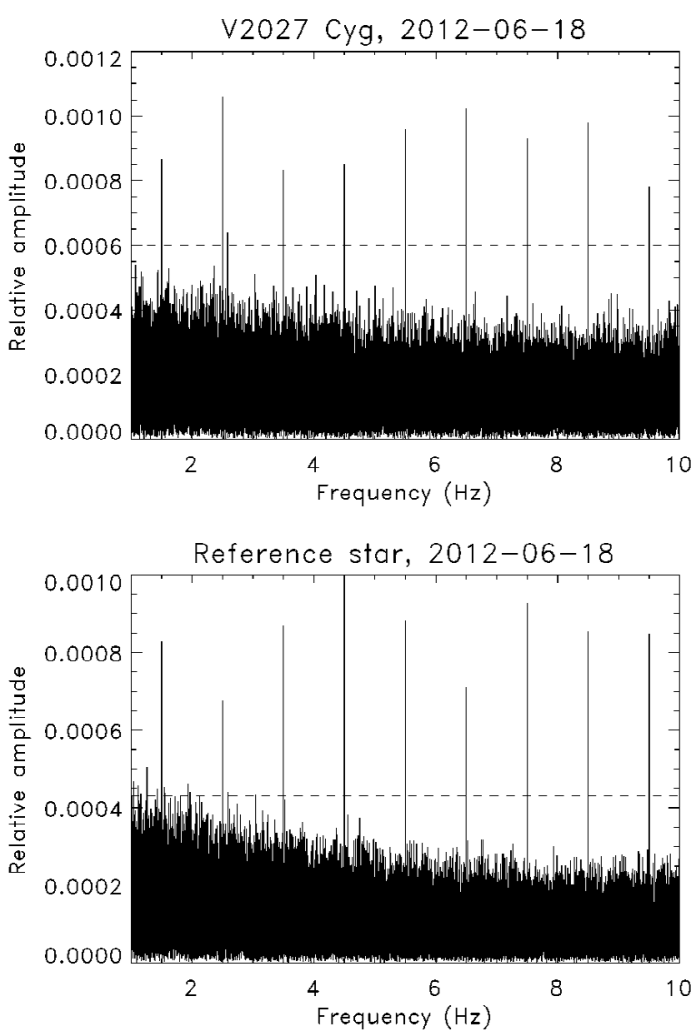

Fig. 6. Fourier relative-amplitude spectra of the data taken on June 18, 2012, with implanted signals.

those two signatures, and because the more severe atmospheric effects at lower frequencies.

\section{Summary and discussion}

Although the multi-fiber photometer Miosotys is designed for the purpose of serendipitous TNO occultation search, its fast photometry makes it useful for detecting possible white dwarf p-mode oscillations. The GW Vir star RX J2117.1+3412 was observed with Miosotys mounted on the 1.93-m telescope at OHP on May 24, 2010, June 17, 2012, and June 18, 2012. One signature at $2.5852 \mathrm{~Hz}$ of $3.5-\sigma$ significance was found in the data taken from June 18, 2012, and another one at $1.2081 \mathrm{~Hz}$ of 3.8- $\sigma$ was found in the combined data of June 17 and 18, 2012. The corresponding relative amplitude of these two signatures are $6.4 \times 10^{-4}$ and $4.7 \times 10^{-4}$, respectively. However, the power spectrum of the reference star shows many peaks around that frequency range, suggesting that atmospheric variations might be the cause of those signatures.

In Fig. 3, we can see the 3- $\sigma$ upper limit to the relative amplitude of non-random fluctuation signals is $6.6,5.3$, and $6.0 \times 10^{-4}$ for the three nights, respectively. If we take a more conservative view to reject the aforementioned two signatures as being real detection, we suggest that a rough estimate of the upper limit to the relative amplitude of any possible oscillations in the frequency range from 1 to $10 \mathrm{~Hz}$ is about $6 \times 10^{-4}$.

We also conducted simulations to examine the coding in our analysis. One example is shown in Fig. 6, where all artificial sinusoidal signals are implanted into the real data at nine half-integer frequencies (in units of $\mathrm{Hz}$ ) with a relative amplitude of $10^{-3}$. The resultant relative amplitudes are not exactly at $10^{-3}$ because of the phase difference between the artificial signal and the original signal (and/or simply random noise) at the corresponding frequency in the data. It is clear that those artificial signals can be easily detected. As expected, the signature at $2.5852 \mathrm{~Hz}$ with a relative amplitude $6.4 \times 10^{-4}$ is not affected by the inclusion of the artificial signals.

It is not clear why the reference star on June 18, 2012, seems to be more affected by atmospheric variations than the target star, V2027 Cyg, in the frequency range of $1-4 \mathrm{~Hz}$. It is similar for the data of June 17, 2012 but to a less extent as seen in Fig. 3. The reference star is about one arcminute away to the south-east of the target star and is actually brighter. Its $V$-band magnitude is 12.0. The transmission efficiency of the fibers used is probably not an issue either, because the recorded intensity of the reference star is higher than the target star and has a higher signal to noise ratio as can be seen from light curves shown in Fig. 1. The reference star on May 24, 2010, is a star of $V$-magnitude 13.6. It is about two arcminutes away to the north of the target star. In Fig. 3, we can see that basically there is no atmospheric effect in the power spectrum above $1 \mathrm{~Hz}$ for both the reference and target star.

We did not perform barycentric time correction to the data because of lacking good knowledge about absolute timing accuracy. It is, however, not so crucial, since the data sets we use to perform Fourier transform are all of two hours or so in time span. The ecliptic longitude and latitude of V2027 Cyg is $(\lambda, \beta)=\left(336.6^{\circ}, 46.9^{\circ}\right)$. The sun was at $\lambda \approx 62.7^{\circ}$ on May 24 , 2010 and at $\lambda \approx 86.7^{\circ}$ on June 17-18, 2012. During these observations, V2027 Cyg is roughly in the direction of the earth's motion but at a high ecliptic latitude. Assuming the earth orbital motion is a circular one with speed $30 \mathrm{~km} \mathrm{~s}^{-1}$, the angle $\theta$ between the earth's motion and the direction to V2027 Cyg is $\cos \theta=0.68$ for May 24, 2010, and $\cos \theta=0.64$ for June $17-$ 18,2012 . The fractional frequency dispersal within two hours due to the earth's motion is about $7 \times 10^{-9}$ for May 24, 2010, and about $3 \times 10^{-8}$ for June $17-18,2012$. The difference in fractional frequency shift between the data sets for May and June is about $4 \times 10^{-6}$. These do not pose any serious problems to the data we currently have, which has a frequency resolution of about $1.5 \times 10^{-4} \mathrm{~Hz}$ only. At this point, we are not able to address the issue of white dwarf oscillation period evolution, which makes the combination of data taken in 2010 and 2012 questionable.

The two signatures at $2.5852 \mathrm{~Hz}$ in the upper right panel of Fig. 3 and at $1.2081 \mathrm{~Hz}$ in the upper panel of Fig. 4, found with data from one night and two consecutive nights, respectively, are intriguing. Future observations with longer data length and better observation conditions will be very much helpful.

Acknowledgements. This work was supported by National Science Council of the Republic of China (Taiwan) under grants NSC 98-2923-M-007-002MY3, 99-2112-M-007-017-MY3, and 101-2923-M-007-001-MY3, and also by Agence National pour la Recherche of France under grant ANR-08-BLAN-0177 Beyond Neptune.

\section{References}

Cósico, A. H., \& Althaus, L. G. 2006, A\&A, 454, 863

Dufour, P., Béland, S., Fontaine, G., Chayer, P., \& Bergeron, P. 2011, ApJ, 733, L19

Fontaine, G., \& Brassard, P. 2008, PASP, 120, 1043

Gautschy, A., Althaus, L. G., \& Saio, H. 2005, A\&A, 438, 1013

Hermes, J. J., Montgomery, M. H., Winget, D. E., et al. 2012, ApJ, 750, L28

Kawaler, S. D. 1993, ApJ, 404, 294

Kawaler, S. D., \& Bradley, P. A. 1994, ApJ, 427, 415

Kawaler, S. D., Bond, H. E., Sherbert, L. E., \& Watson, T. K. 1994, AJ, 107, 298

Landolt, A. U. 1968, PASP, 153, 151 
H.-K. Chang et al.: P-mode oscillations in RX J2117.1+3412

Montgomery, M. H., Williams, K. A., Winget, D. E., et al. 2008, ApJ, 678, L51 Motch, C., Werner, K., \& Pakull, M. W. 1993, A\&A, 268, 561

Ostriker, J. P. 1971, ARA\&A, 9, 353

Quirion, P.-O., Fontaine, G., \& Brassard, P. 2004, ApJ, 610, 436

Rauch, T., \& Werner, K. 1997, in The Third Conference on Faint Blue Stars, eds. A. G. D. Philip, J. Liebert, \& R. A. Saffer (Schenectady, NY: L. Davis Press), 217

Robinson, E. L. 1984, AJ, 89, 1732

Saio, H., Winget, D. E., \& Robinson, E. L. 1983, ApJ, 265, 982
Shih, I.-C. et al. 2010, in Ground-based and Airborne Instrumentation for Astronomy III, eds. I. S. McLean, S. K. Ramsay, \& H. Takami, Proc. of SPIE 7735,773546

Silvotti, R., Fontaine, G., Pavlov, M., et al. 2011, A\&A, 525, A64

Starrfield, S., Cox, A. N., Hodson, S. W., \& Clancy, S. P. 1983, ApJ, 269, 645

van der Klis, M. 1989, in Timing Neutron Stars, NATO ASI C262, 27

Vauclair, G., Moskalik, P., Pfeiffer, B., et al. 2002, A\&A, 381, 122

Werner, K., Dreizler, S., Heber, U., et al. 1996, A\&A, 307, 860

Winget, D. E., \& Kepler, S. O. 2008, ARA\&A, 46, 157 